\title{
Effect of Different Strength of Medium on Organogenesis, Phenolic Accumulation and Antioxidant Activity of Spearmint (Mentha spicata 1.)
}

\author{
Dani Fadel ${ }^{1}$, Spiridon Kintzios ${ }^{*}, 2$, Athanasios S. Economou ${ }^{1}$, Georgia Moschopoulou ${ }^{2}$ and \\ Helen-Isis A. Constantinidou ${ }^{3}$ \\ ${ }^{I}$ Department of Horticulture, School of Agriculture, Aristotle University, 54124 Thessaloniki, Greece \\ ${ }^{2}$ Laboratory of Plant Physiology, Faculty of Agricultural Biotechnology, Agricultural University of Athens, 11855 \\ Athens, Greece \\ ${ }^{3}$ Department of Crop Science, School of Agriculture, Aristotle University 54124 Thessaloniki, Greece
}

\begin{abstract}
We investigated the effect of inorganic salt concentration on the in vitro organogenesis of spearmint (Mentha spicata L.) by using Murashige and Skoog culture medium of different strength (full, half- and quarter strength). The highest number of shoots and roots induced per explant (3.5 and 10, respectively), as well as the maximum average shoot length $(16 \mathrm{~cm})$ was observed on half strength medium. The maximum leaf number (35 per explant) and average root length $(7 \mathrm{~cm})$ were associated with regeneration on full medium. The highest average phenolic content was observed on shoot extracts $(7.20 \mathrm{mg} / \mathrm{g}$ f.w.) and root extracts $(5.93 \mathrm{mg} / \mathrm{g}$ f.w.) cultured onto quarter and half strength medium, respectively. The strength of the culture medium was inversely correlated with the antioxidant activity of plant extracts. Therefore, for spearmint organogenesis, half strength MS medium offers a compromise between optimum growth in vitro and antioxidant phenolic accumulation.
\end{abstract}

Keywords: Antioxidant activity, medium strength, Mentha spicata, Murashige and Skoog basal medium, phenolics.

\section{INTRODUCTION}

Mentha is a genus of aromatic perennial herbs belonging to the family of Lamiaceae. It is distributed mainly in the temperate and sub-temperate regions of the world. Mentha has a large number of species that differ widely in their characteristics and polyploidy level. It is known to comprise about forty recognizable species, among them spearmint (Mentha spicata L.), a hybrid of $M$. longifolia and $M$. rotundifolia. As a hybrid, spearmint is rarely propagated by seeds and as a consequence, is exclusively propagated from its vegetative parts and by micropropagation. Spearmint has many uses in foodstuff, flavours, beverages, cosmetics and folks medicine [1-3].

In the past, protocols for plantlet regeneration from spearmint explants such as axillary buds and leaves have been reported and the effect of different nutrient media and growth regulators was investigated [2,4]. Minerals are important components of the culture medium. There is a large choice of combinations of macro- and micro-salt mixtures. The most widely used culture medium is described in Murashige and Skoog [5] (MS medium), because most plant cell cultures react to it favorably. It is classified as a high salt medium in comparison to many other formulations, with high levels of nitrogen, potassium and some of the micronutrients, particularly boron and manganese [6]. Due to its

*Address correspondence to this author at the Laboratory of Plant Physiology, Faculty of Agricultural Biotechnology, Agricultural University of Athens, 11855 Athens, Greece; Tel: +302105294292; Fax: +302105294286; E-mail: skin@aua.gr high salt content, however, this nutrient medium is not necessarily always optimal for growth and development of explants and plantlets in vitro [7].

In the present study, we investigated the effects of the strength of solid MS medium (MS, $1 / 2$ MS and $1 / 4 \mathrm{MS}$ ) on the organogenesis, total phenolic content and antioxidant activity of shoot and root extracts from regenerated spearmint plantlets.

\section{MATERIALS AND METHODS}

\section{Explant Preparation}

Explants were derived from apical shoot segments carrying 4-5 nodes, which were separated from a single six-month old spearmint plant, surface sterilized with $0.1 \%(\mathrm{w} / \mathrm{v})$ $\mathrm{HgCl}_{2}$ with 2-3 drops of Tween 20 for 2 minutes followed by thorough washing in sterile distilled water. Shoots were cut into $1.5-2 \mathrm{~cm}$ long pieces, each carrying a node.

\section{Shoot and Root Formation}

For shoot proliferation, the nodal segments were incubated for four weeks placed vertically in MS media of various basal strengths, i.e. containing inorganic salts at various concentrations ( $1 / 4 \mathrm{MS}, 1 / 2 \mathrm{MS}$ and full strength $\mathrm{MS})$ with $3 \%(\mathrm{w} / \mathrm{v})$ sucrose, $0.8 \%(\mathrm{w} / \mathrm{v})$ agar, $0.1 \%(\mathrm{w} / \mathrm{v})$ myoinositol and supplemented with $0.2 \mathrm{mg} / \mathrm{L}$ 1-naphtalene acetic acid (NAA) and $1 \mathrm{mg} / \mathrm{L}$ kinetin. The $\mathrm{pH}$ was adjusted to 5.8 . Cultures were maintained at $25 \pm 2^{\circ} \mathrm{C}$ under a photosynthetic photon flux density of $90 \mu \mathrm{mol} \mathrm{m} \mathrm{m}^{-2} \mathrm{~s}^{-1}$ (16 h of light daily, provided from cool white fluorescent lamps). 
At the end of the four-week culture period, regenerated shoots were separated and individually transferred for two weeks for rooting to growth regulator-free MS medium of similar basal strength to shoot proliferation medium. Separated shoot and root segments from 50 regenerated plantlets, randomly chosen from each MS strength medium (1/4 MS, 1/2 MS, full MS), were then subjected to organogenesis evaluation and phytochemical analysis.

\section{Evaluation of Organogenesis}

Shoot formation was evaluated in terms of average shoot length $(\mathrm{cm})$ and shoot number per explant and average shoot length per regenerant. The number of leaves per culture was determined by counting the fully developed green leaves formed on each plantlet and then determining the mean number of leaves per plantlet for each treatment. The number of roots was determined by counting the number of roots ( $>$ $0.5 \mathrm{~cm}$ length) formed after transfer of adventitious shoots to the rooting media.

\section{Biochemical Assays}

Extraction of phenolics was performed according to procedure described by Kintzios et al. [8]. Shoots and roots were weighted separately (mg fresh weight) and macerated for 5 minutes in $10 \mathrm{~mL} \mathrm{MeOH} 80 \%(v / v)$. The extract was then placed into a water bath at $80^{\circ} \mathrm{C}$ for 30 minutes, filtrated through Whatman filter paper, collected in $10 \mathrm{~mL}$ tubes and stored at $-20^{\circ} \mathrm{C}$ for analysis. The total phenolic content was determined spectrophotometrically at $333 \mathrm{~nm}$ and expressed by means of $\mathrm{mg}$ rosmarinic acid equivalent per gram fresh weight.

The free-radical scavenging capacity of the phenolic extracts from shoot and root segments of the 150 regenerated spearmint plantlets produced on MS media as previously described (50 on each different medium), was evaluated with the DPPH stable radical following a methodology originally described by Blois [9] and modified by Jao and Ko [10]. Briefly, to $2 \mathrm{~mL}$ of DPPH in $80 \% \mathrm{MeOH}(0.1 \mathrm{mM}) 0.9 \mathrm{~mL}$ tris $\mathrm{HCl}(50 \mathrm{mM}, \mathrm{pH} 7.4)$ were added plus either $0.1 \mathrm{~mL}$ aliquot extract or methanolic solution (as control). Following a 30 minute incubation in darkness, the absorbance was measured at $517 \mathrm{~nm}$. A lower absorbance of the reaction mixture indicates a higher activity in scavenging free radicals.

The percentage reduction of the DPPH, referred as "inhibition" or "quenching" or "scavenging" and defined by $\mathrm{Q}=100\left(\mathrm{~A}_{0}-\mathrm{A}_{1}\right) / \mathrm{A}_{0}$, was calculated, where $\mathrm{A}_{0}$ is the initial absorbance and $A_{1}$ is the value for the extract. The results were presented as values of $\mathrm{IC}_{50}$, which is the concentration of the antioxidants tested where $50 \%$ scavenging of the free radicals was obtained. The lower the $\mathrm{IC}_{50}$ number, the greater is the overall effectiveness of the antioxidant in question, namely the phenolic extracts.

\section{Statistical Analysis}

Experiments were set-up in a completely randomized design. Each experiment was repeated twice. Statistical analysis was based on analysis of variance (ANOVA). Significant differences $(p<0.01)$ among the means were determined by Duncan's multiple range test. Correlations between determined organogenesis and biochemical parameters were calculated using MS-Excel software.

\section{RESULTS AND DISCUSSION}

\section{Effect of the Medium Strength on Shoot and Root Formation}

Four weeks of incubation of spearmint nodal explants onto $1 / 4 \mathrm{MS}, 1 / 2 \mathrm{MS}$ and full MS medium supplemented with $0.2 \mathrm{mg} / \mathrm{L} 1$-naphtalene acetic acid (NAA) and $1 \mathrm{mg} / \mathrm{L} 1$ kinetin resulted into the formation and growth of adventitious shoots. Roots were developed two weeks after the transfer of individual shoots on growth regulator-free $1 / 2 \mathrm{MS}$ and full MS media. No roots were induced on $1 / 4$ MS. A significant effect of the strength of the culture medium on shoot, leaf and root formation was observed (Table 1). The maximum number of shoots and roots induced per explant (3.5 and 10, respectively), as well as the maximum average shoot length $(16 \mathrm{~cm})$ was observed on half-strength medium supplemented with $0.2 \mathrm{mg} / \mathrm{L} \mathrm{NAA}$ and $1 \mathrm{mg} / \mathrm{L}$ kinetin. The maximum leaf number (35 per explant) and average root length $(7 \mathrm{~cm})$ was associated with regeneration on full MS medium. The length and the number of shoots were positively correlated with each other $\left(r^{2}=0.915, p<0.05\right)$. In the rooting phase, adventitious shoots cultured on $1 / 2$ MS produced almost the double number of roots (although shorter) than when grown on full MS medium. Halving the strength of MS medium resulted in increased rooting of Mentha arvensis regenerants [11]. Tetsumura et al. [12] also observed that a reduction in the strength of MS medium resulted in the increase of in vitro shoot and root formation from highbush blueberry (Vaccinium corymbosum and $V$. virgatum). Other researchers have also reported the beneficial effect of a reduction of the strength of the culture medium on the in vitro initiation of roots of plant species such as rose [13] and globe artichoke [14-16]. Patel and Shah [17] reported that root number and root length of Stevia rebaudiana plant cultures were significantly influenced by the strength of MS medium ( $1 / 4 \mathrm{MS}, 1 / 2$ MS and full strength MS) and treatment combinations of IBA, NAA and BAP. Such combinations may have affected cell differentiation

Table 1. Effect of the Strength of the Solid Culture Medium on Shoot, Leaf and Root Formation. Numbers Followed by Different Letters Indicate Statistically Different Values $(\mathbf{p}<\mathbf{0 . 0 1})$. Each Number is an Average of 50 Observations

\begin{tabular}{|c|c|c|c|c|c|}
\hline \multirow{2}{*}{ Medium Strength } & \multicolumn{2}{|c|}{ SHOOT } & \multirow{2}{*}{$\begin{array}{c}\text { LEAF } \\
\text { Number }\end{array}$} & \multicolumn{2}{|c|}{ ROOT } \\
\hline & Number & Length (cm) & & Number* & Length (cm) \\
\hline $1 / 4 \mathrm{MS}$ & $2.10 \pm 0.19^{b}$ & $8.03 \pm 0.45^{\mathrm{c}}$ & $34.78 \pm 2.68^{\mathrm{ab}}$ & - & - \\
\hline Full MS & $3.26 \pm 0.42^{\mathrm{a}}$ & $11.78 \pm 0.37^{b}$ & $35.1 \pm 2.98^{\mathrm{a}}$ & $5.06 \pm 0.44^{\mathrm{b}}$ & $7.06 \pm 0.37^{\mathrm{a}}$ \\
\hline
\end{tabular}

*Only roots longer than $0.5 \mathrm{~cm}$ were counted. 
and elongation. Moreover, Villamor [18] conducted a similar experiment but to determine the effects of media strength (full MS, $1 / 2 \mathrm{MS}$ ) and sources of nitrogen $\left(\mathrm{NH}_{4} \mathrm{NO}_{3}, \mathrm{KNO}_{3}\right.$ ) on shoot (number, length), leaf (number, length, width) and root number of ginger. The results were similar to the ones obtained in our experiment and indicated an increase of root number with the dilution of MS basal medium and the concomitant decrease of shoot number, shoot length, leaf number. On the contrary, Jain et al. [19] found a direct association between medium strength and the number and length of shoots induced from Harpagophytum procumbens tissue cultures. The effect of the medium strength could be possibly associated with particular components of the culture medium. For example, even minor changes in the concentration of trace elements can dramatically affect plant organogenesis in vitro. Castiglione et al. [20] observed a radical reduction in the number and length of roots induced from white poplar (Populus alba) cultures when zinc concentration in the culture medium was increased.

\section{Effect of the Medium Strength on Total Phenolic Content and Antioxidant Activities of Shoot and Root Extracts}

The highest average phenolic content (in terms of equivalent rosmarinic acid concentration) in regenerated spearmint tissues was found to be $7.20 \pm 0.39 \mathrm{mg} / \mathrm{g}$ f.w. from shoot extracts cultured onto $1 / 4$ MS and $5.93 \pm 0.84 \mathrm{mg} / \mathrm{g}$ f.w. from root extracts cultured onto $1 / 2$ MS (Table 2).

The effect of different nutrient media and growth regulators on spearmint regeneration has been previously investigated [2,4]. Recently, Tisserat and Vaughn [21] demonstrated the potential use of in vitro cultures of Mentha spicata L. for essential oil production. The present study is the first report demonstrating the possible effect of MS strength on the total
Table 2. Effect of the Strength of the Solid Culture Medium on the Accumulation of total Phenolics (Expressed as Equivalent Rosmarinic Acid Concentration (mg/g f.w. \pm Standard Error) in Shoot and Root Extracts from Spearmint Regenerants. Numbers Followed by Different Letters Indicate Statistically Different Values $(\mathbf{p}<0.01)$. Each Number is an Average of 50 Observations

\begin{tabular}{|c|c|c|}
\hline Medium Strength & Shoot Extracts & Root Extracts \\
\hline \hline $1 / 4 \mathrm{MS}$ & $7.20 \pm 0.39^{\mathrm{a}}$ & - \\
\hline $1 / 2 \mathrm{MS}$ & $3.48 \pm 0.51^{\mathrm{b}}$ & $5.93 \pm 0.84^{\mathrm{a}}$ \\
\hline Full MS & $1.98 \pm 0.23^{\mathrm{c}}$ & $2.20 \pm 0.31^{\mathrm{b}}$ \\
\hline
\end{tabular}

phenolic content in spearmint shoot and root extracts. The inverse relationship between medium strength and secondary metabolite accumulation in spearmint tissue cultures could be possibly explained by the fact that full nutrient media predominantly promote primary metabolism and cellular growth, in some cases hampering morphological and biochemical tissue differentiation. This hypothesis has been previously verified for the inhibition of differentiation of melon callus cultures by increased concentrations of soluble carbohydrates and selected macronutrient ions $\left(\mathrm{K}^{+}, \mathrm{Ca}^{2+}\right)$ [22]. E1Keltawi and Croteau [23] have previously demonstrated on a whole-plant level that increased salinity causes a depression of growth and essential oil formation in spearmint, perhaps via inhibition of cytokinin biosynthesis and transport. Hirata et al. [24] also observed a reduction in total essential oil concentration when spearmint shoot tip cultures were transferred from Gamborg's B5 medium [25] to the richer MS medium.

A

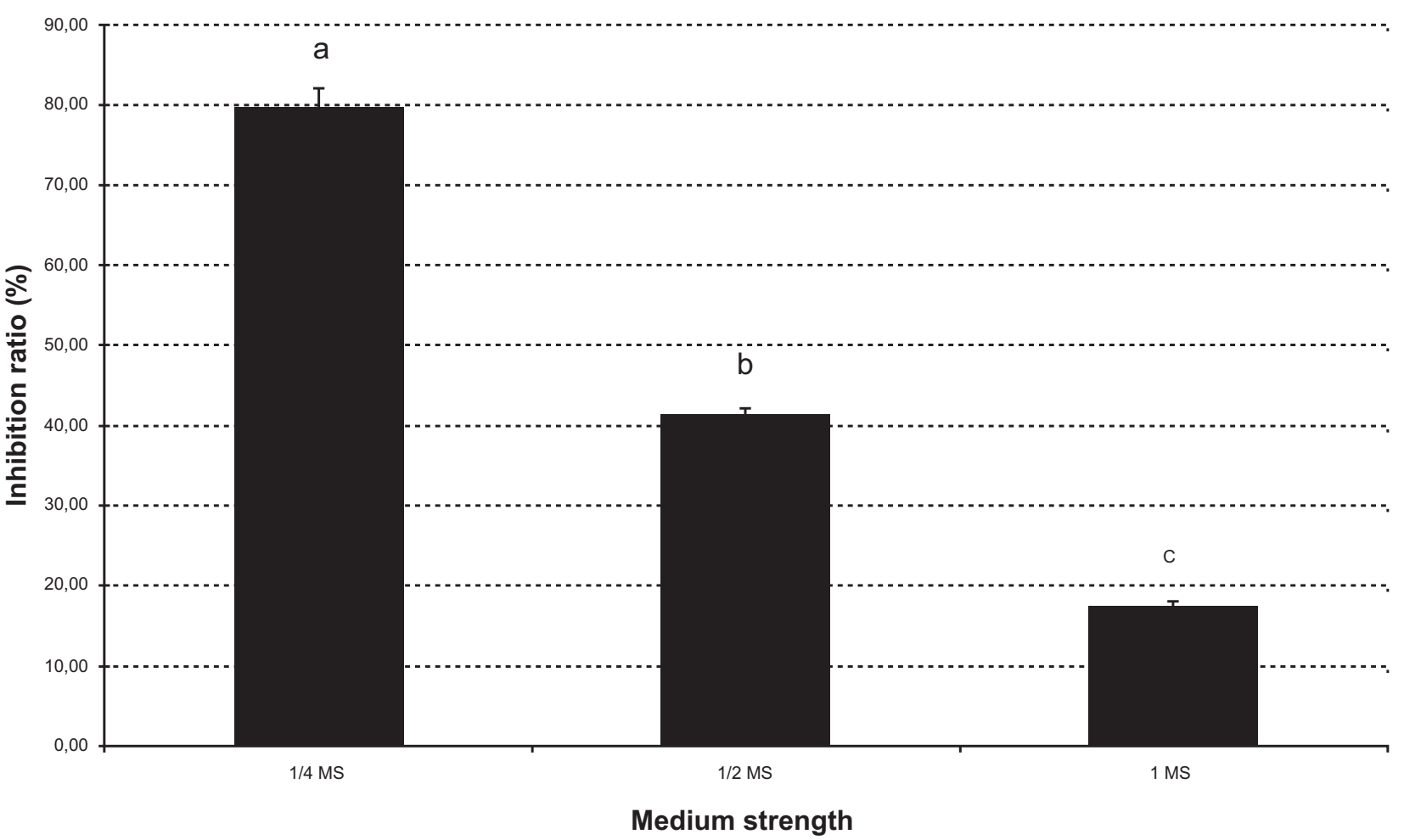


(Fig. 1) Contd.....

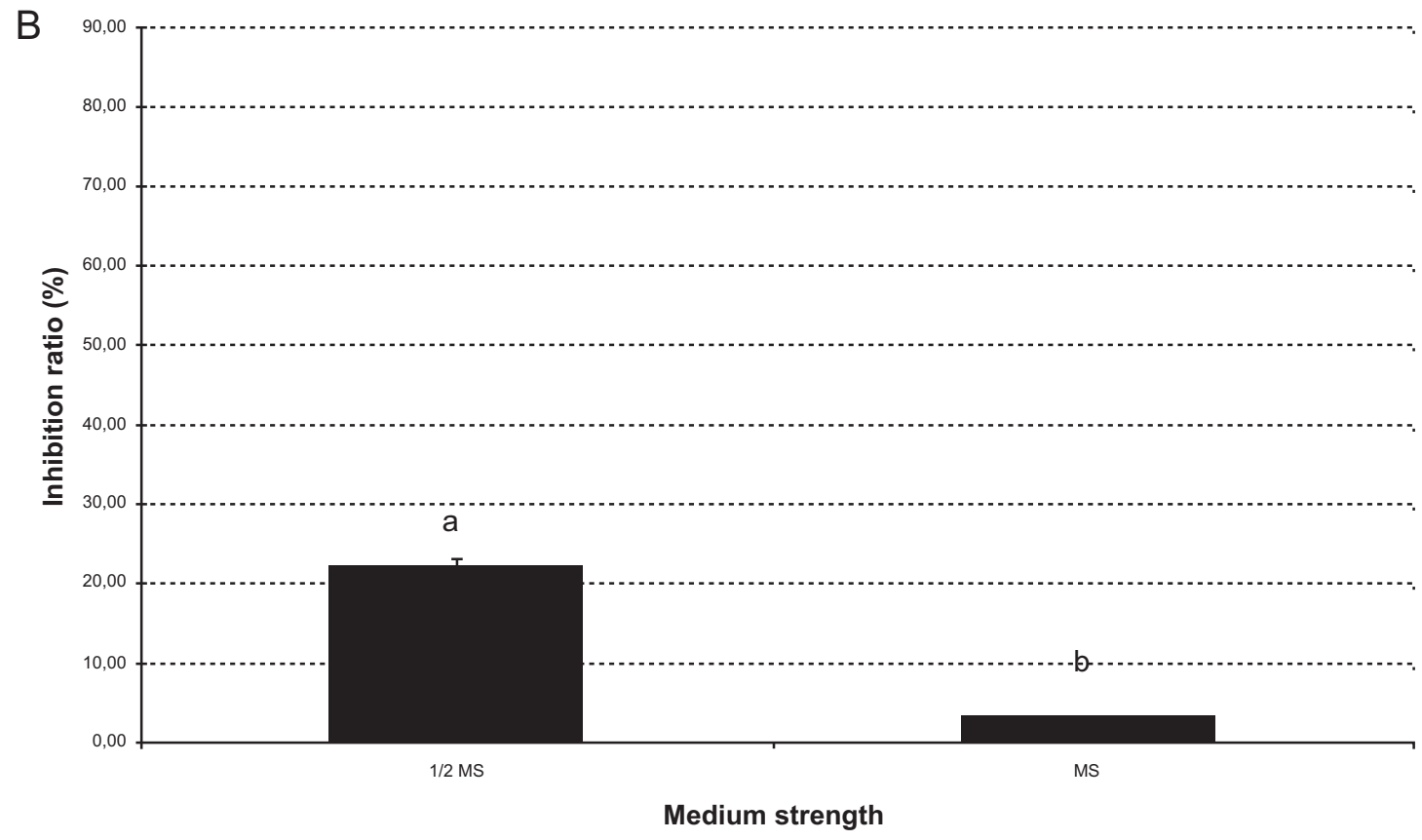

Fig. (1). Antioxidant activity (expressed as \% inhibition of DPPH) of shoot (A) and root (B) extracts from spearmint regenerant groups, grown on $1 / 4 \mathrm{MS}, 1 / 2 \mathrm{MS}$ and full strength MS media. Columns marked with different letters indicate statistically different values $(\mathrm{p}<0.01)$.

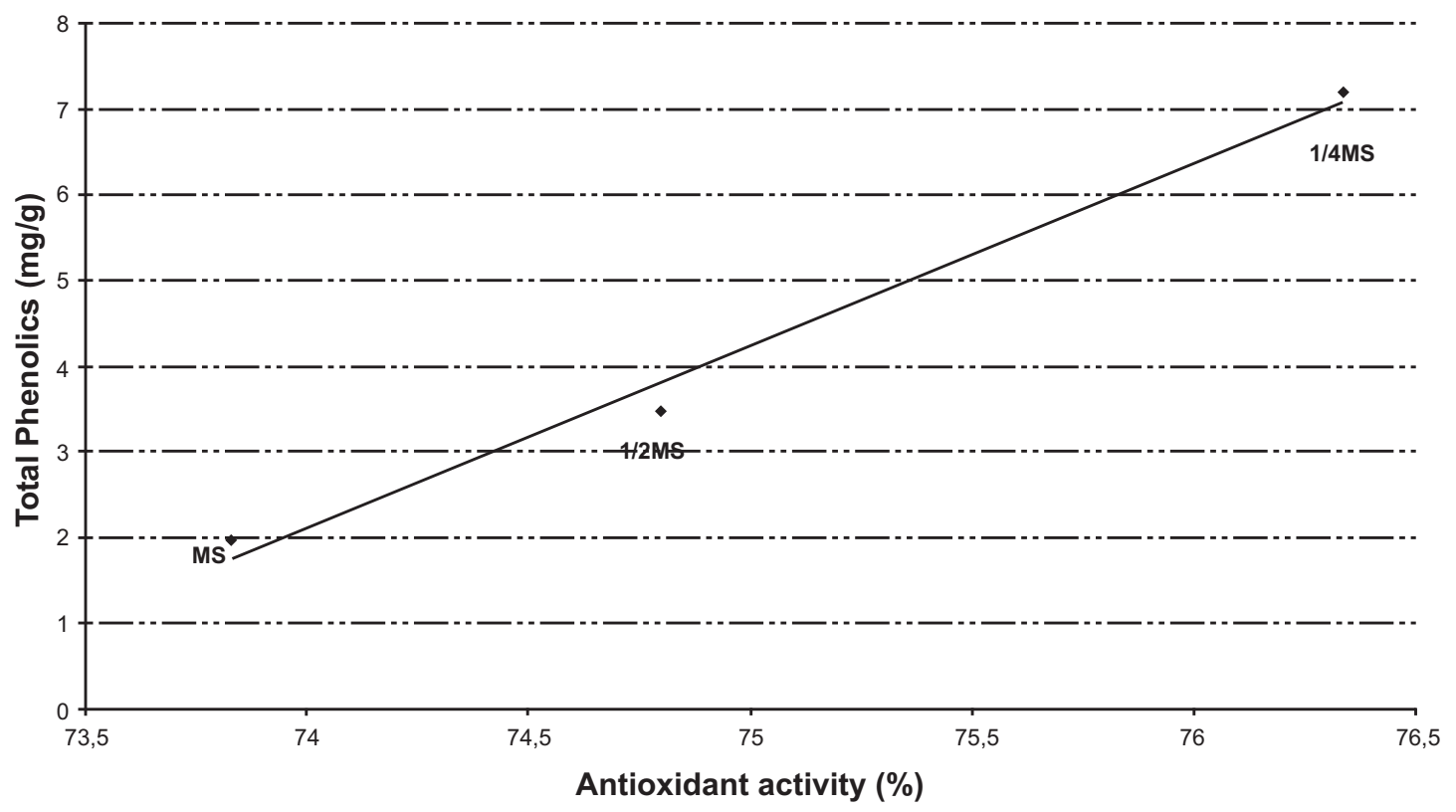

Fig. (2). Relationship between average total phenolic content (expressed as equivalent rosmarinic acid concentration) and DPPH' scavenging activity of extracts derived from spearmint adventitious shoots grown on $1 / 4 \mathrm{MS}, 1 / 2 \mathrm{MS}$ and full MS media.

The DPPH-inhibitory activity of adventitious shoot extracts was decreased by increasing the culture medium strength from $1 / 4$ to $1 / 2$ to full strength (Fig. 1A). The same pattern was observed for root extracts, although their antioxidant potency was considerably lower than that of shoot extracts (Fig. 1B). The high positive correlation between the total phenolic content and DPPH radical-scavenging $\left(\mathrm{r}^{2}=\right.$ $0.99, \mathrm{p}<0.05)$ in the extracts of adventitious shoots grown on $1 / 4 \mathrm{MS}, 1 / 2 \mathrm{MS}$ and full strength MS media is shown in Fig. (2). Other researchers have also reported a high positive correlation between free radical-scavenging activity and the total concentration of phenolic compounds in plant extracts [26, 27]. Tawaha et al. [28] observed a positive correlation between the antioxidant activity and total phenolic content for methanolic extracts of 51 Jordanian plant species, including $M$. spicata. 


\section{CONCLUSION}

The results of the present study indicate that, for spearmint regeneration, half strength MS medium offers a compromise between optimum growth in vitro and antioxidant phenolic accumulation. Variation of medium composition could lead to enhanced spearmint regeneration efficiency with a lower cost (due to lower concentration of medium constituents). At the same time, the phytochemical quality of regenerated plantlets could be increased due to their higher content in phenolic compounds with antioxidant activity (provided, that this trait is maintained ex vitro).

\section{ACKNOWLEDGEMENTS}

Dani Fadel acknowledges the support of the Greek National Scholarship Foundation.

ABBREVIATIONS
$1 / 4 \mathrm{MS}=\begin{aligned} & \text { Quarter strength Murashige and Skoog } \\ & \text { medium }\end{aligned}$
$1 / 2 \mathrm{MS}=$ Half strength Murashige and Skoog medium
$\mathrm{MS}=$ Full strength Murashige and Skoog medium
$\mathrm{DPPH}=2,2$-diphenyl-1-picrylhydrazyl

\section{REFERENCES}

[1] Li X, Niu X, Bressan RA, Weller SC, Hasegawa PM. Efficient plant regeneration of native spearmint (Mentha spicata $\mathrm{L}$.). In vitro Cell Dev Biol Plant 1999; 35: 333-8.

[2] Bhat S, Maheshwari P, Kumar S, Kumar A. Mentha species: In vitro regeneration and genetic transformation. Mol Biol Today 2002; 3: 11-23.

[3] Kanatt SR, Chander R, Sharma A. Antioxidant potential of mint (Mentha spicata L.) in radiation-processed lamb meat. Food Chem 2007; 100: 451-8.

[4] Shasany AK, Khanuja SPS, Dhawan S, Yadav U, Sharma S, Kumar S. High regenerative nature of Mentha arvensis internodes. J Biosci 1998; 23: 641-6.

[5] Murashige T, Skoog F. A revised method for rapid growth and bioassays with tobacco tissue cultures. Physiol Plant 1962; 15: 47297.

[6] Cohen D. The culture medium. Acta Hort 1995; 393: 15-24.

[7] Pierik RLM. In vitro Cultures of higher plants. Dordrecht, The Netherlands: Martinus Nijhoff Publishers 1997.

[8] Kintzios S, Makri O, Panagiotopoulos E, Scapeti M. In vitro rosmarinic acid accumulation in sweet basil (Ocimum basilicum L.). Biotechnol Lett 2003; 25: 405-8.
[9] Blois MS. Antioxidant determination by the use of a stable free radical. Nature 1958; 181: 1199-200.

[10] Jao CH, Ko WC. 1,1-diphenyl-1-picrylhydrazyl (DPPH) radical scavenging by protein hydrolysates from tuna cooking juice. Fishery Sci 2002; 68: 430-5.

[11] Phatak SV, Heble MR. Organogenesis and terpenoid synthesis in Mentha arvensis. Fitoter 2002; 73: 32-9.

[12] Tetsumura T, Matsumoto Y, Sato M, et al. Evaluation of basal media for micropropagation of four highbush blueberry cultivars. Sci Hort 2008; 119: 72-4.

[13] Sauer A, Walther F, Preil W. Different suitability for in vitro propagation of rose cultivars. Gartenbauwiss 1985; 3: 133-8.

[14] Ancora G. Globe artichoke (Cynara scolymus L.). In: Biotechnology in agriculture and forestry, Ed. Y.P.S. Bajaj, Berlin: Springer-Verlag 1986; 2: pp. 471-86.

[15] Iapichino G. Micropropagation of globe artichoke (Cynara scolymus L.) from underground dormant buds ("ovoli"). In vitro Cell Dev Biol Plant 1996; 32: 249-52.

[16] Lauzer D, Vieth J. Micropropagation of seed-derived plant of Cynara scolymus L., cv. 'Green globe'. Plant Cell Tiss Org Cult 1990; 21: 237-44.

[17] Patel RM, Shah RR. Regeneration of Stevia plant through callus culture. Indian J Pharm Sci 2009; 71: 46-50.

[18] Villamor CC. Influence of media strength and sources of nitrogen on micropropagation of ginger, Zingiber officinale Rosc. E-Int Sci Res J 2010; 2: 150-55.

[19] Jain N, Bairu MW, Stirk WA, Van Staden J. The effect of medium, carbon source and explants on regeneration and control of shoot-tip necrosis in Harpagophytum procumbens. South Afr J Bot 2008; 75: 117-21.

[20] Castiglione S, Franchin C, Fossati T, Lingua G, Torrigiani P, Biondi. S. High zinc concentrations reduce rooting capacity and alter metallothionein gene expression in white poplar (Populus alba L. cv. Villafranca). Chemosphere 2007; 67: 1117-26.

[21] Tisserat B, Vaughn SF. Growth, morphogenesis, and essential oil production in Mentha spicata L. plantlets in vitro. In vitro Cell Dev Biol Plant 2008; 44: 40-50.

[22] Kintzios S, Stavropoulou Er, Skamneli S. Accumulation of selected macronutrients and carbohydrates in melon tissue cultures: association with pathways of in vitro dedifferentiation and differentiation (organogenesis, somatic embryogenesis). Plant Sci 2004; 167: 655-64.

[23] El-Keltawi NE, Croteau R. Salinity depression of growth and essential oil formation in spearmint and marjoram and its reversal by foliar applied cytokinin. Phytochemistry 1987; 26: 1333-4.

[24] Hirata T, Murakami S, Ogihara K, Suga T. Volatile monoterpenoid constituents of the plantlets of Mentha spicata produced by shoot tip culture. Phytochemistry 1990; 29: 493-5.

[25] Gamborg OL, Miller RA, Ojima K. Nutrient requirements of suspension cultures of soybean root cells. Exp Cell Res 1968; 50: 151-8.

[26] Wangensteem H, Samuelsen AB, Malterud KE. Antioxidant activity in extracts from coriander. Food Chem 2004; 88: 293-7.

[27] Zheng W, Wang SY. Antioxidant activity and phenolic compounds in selected herbs. J Agric Food Chem 2001; 49: 5165-70.

[28] Tawaha K, Alali FQ, Gharaibeh M, Mohammad M, El-Elimat T. Antixoidant activity and total phenolic content of selected Jordanian plant species. Food Chem 2007; 104: 1372-8. 